\section{A method for movement}

Sally H. Zigmond

\section{During cell movement, microtubules may control the actin cytoskeleton through specific Rho GTPases. An understanding of the spatial control of cell motility may now be within reach.}

$C_{\mathrm{t}}^{\mathrm{e}}$ ell movement is a highly coordinated process. First, the front of the cell protrudes and then attaches to the substratum on which the cell is moving. Then, the cytoplasm moves forward. Finally, the cell's rear releases its attachments to the substratum and moves forward. All of these steps depend on localized polymerization of the cytoskeletal protein actin, which forms different structures in different parts of the motile cell. Specific active GTP-hydrolysing proteins (GTPases) of the Rho family ${ }^{1,2}$ can induce these processes.

Microtubule dynamics can also coordinate actin-based movements - for example, when microtubules depolymerize, a cell loses polarity and its migration speed slows $^{3-5}$. Are Rho GTPases, microtubule dynamics and actin polymerization linked? Studies of the Rho proteins Rho and Rac by Ren and colleagues ${ }^{6}$ and Waterman-Storer

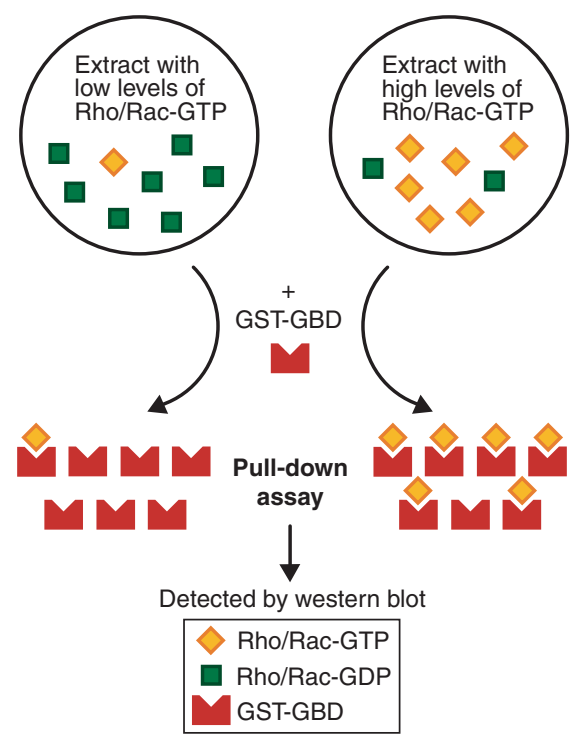

Figure 1 The 'pull-down' assay used to detect active Rac and $R o^{6,7}$. The assay uses the G-protein-binding domain (GBD) of a downstream target of Rac or Rho that selectively binds the Rho protein in its active (GTP-bound) form. The GBD is fused to glutathione-S-transferase (GST). Binding of the GBD to Rac or Rho is stable enough to allow isolation of the GBD-Rac/Rho-GTP complex. The precipitated GTPase is then quantified by a western blot. and co-workers ${ }^{7}$ now suggest an answer.

If we want to understand exactly how Rho GTPases could be linked to microtuin the cell they are active. Rho proteins are active when they bind GTP, and inactive when they bind GDP. So we need to know how many of the proteins are in their GTPbound form when known microtubule and actin dynamics are taking place. For this purpose, Waterman-Storer et al. ${ }^{7}$ and Ren et al. ${ }^{6}$ have used a new 'pull-down' assay (Fig. 1).

The new assay of Rho activation ${ }^{6}$ helps to explain how microtubule depolymerization decreases cell movement and increases the contractile force that cells impart to the substratum $^{3,5}$. It was thought that microtubule depolymerization might increase force by merely removing an internal mechanical constraint on contraction ${ }^{8}$. Alternatively, depolymerization might stimulate contraction of actin-myosin chains, as increased phosphorylation of the myosin light chain follows microtubule depolymerization". Rho stimulates this phosphorylation and inhibitors of Rho block both the increased force and the phosphorylation ${ }^{10,11}$. Now Ren et al. ${ }^{6}$ show that induction of microtubule depolymerization by the drug colchicine in 3T3 fibroblasts activates Rho, as determined in a pull-down assay; active Rho then stimulates cytoplasmic contraction ${ }^{9-11}$.

Active Rac, in contrast, can induce the formation of cell protrusions, and so enhances cell motility. Microtubule dynamics can also modulate cell protrusions, and Waterman-Storer et al..$^{7}$ reveal how Rac and microtubules can be linked to enhance actin polymerization and the formation of protrusions. Protrusion at the cell surface decreases following depolymerization of microtubules by the drug nocodazole $e^{4}$. Waterman-Storer et al. ${ }^{7}$ show that when nocodazole is washed out and microtubules reassemble, protrusive activity increases. It seems not to be the amount of polymerized microtubules that is important, but rather their dynamics - the changes between periods of microtubule depolymerization and polymerization. If microtubule dynamics are blocked, cell migrations and surface protrusions are inhibited ${ }^{12-14}$.

Are the effects of these microtubule dynamics on actin polymerization mediated through Rac? The answer is probably bule dynamics and actin polymerization, we need to be able to assay when and where 'yes' - Waterman-Storer et al. ${ }^{7}$ show that dominant-negative Rac blocks the increased protrusion induced by microtubule dynamics, and that microtubule polymerization increases the amount of active Rac detected in a pull-down assay. Their studies of Rac are also consistent with the idea that it is the microtubule dynamics, rather than the amount of microtubules, that are important.

It isn't clear how microtubule dynamics modulate the levels of active Rho and Rac. One clue is that microtubules co-localize with several guanine-nucleotide-exchange factors with activities for Rho and $\mathrm{Rac}^{15,16}$ these exchange proteins control whether Rho and Rac are active or inactive. Rac itself also associates with tubulin and/or microtubules ${ }^{7}$, as do other proteins that may indirectly regulate Rho or Rac.

Different microtubule dynamics, therefore, increase levels of active Rho or Rac, and Rho and Rac in turn have different effects on cell motility. Might the effects of microtubule dynamics, acting through Rac and Rho, also act at a subcellular level - to coordinate the different processes that go on in different parts of the cell during movement? The cell front contains the highest density of dynamically unstable microtubule ends ${ }^{7}$, and Waterman-Storer et al. propose that this dynamic instability, acting through Rac, is what causes protrusion to localize at the front. Further back in the cell, net microtubule depolymerization ${ }^{17}$ might locally activate Rho and increase cytoplasmic contractility ${ }^{18}$. It seems possible that, in large cells, microtubule dynamics can help coordinate cell motility by modulating Rho and Rac.

Sally H. Zigmond is at the Biology Department,

University of Pennsylvania, 415 South University

Ave, Philadelphia, Pennsylvania 19104-6018, USA.

e-mail: szigmond@sas.upenn.edu

1. Ridley, A. et al. Cell 70, 401-410 (1992).

2. Nobes, C. D. \& Hall, A. J. Cell Biol. 144, 1235-1244 (1999).

3. Danowski, B. J. Cell Sci. 93, 255-266 (1989).

4. Bershadsky, A. D., Vaisberg, E. A. \& Vasiliev, J. M. Cell Motil. Cytoskeleton 19, 152-158 (1991).

5. Bershadsky, A., Chausovsky, A, Becker, E., Lyubimova, A. \& Geiger, B. Curr. Biol. 6, 1279-1289 (1996).

6. Ren, X.-D., Kiosses, W. B. \& Schwartz, M. A. EMBO J. 18, 578585 (1999).

7. Waterman-Storer, C. M., Worthylake, R. A., Liu, B. P., Burridge, K. \& Salmon, E. D. Nature Cell Biol. 1, 45-50 (1999).

8. Ingber, D. E. J. Cell Sci. 104, 613-627 (1993).

9. Kolodney, M. \& Elson, E. Proc. Natl Acad. Sci. USA 92, 1025210256 (1995).

10. Enomoto, T. Cell Struct. Funct. 21, 317-326 (1996).

11. Liu, B.P., Chrzanowske-Wodnicka, M. \& Burridge, K. Cell Adhesion Commun. 5, 249-255 (1998).

12. Tanaka, E., Ho, T \& Kirschner, M. W. J. Cell Biol. 128, 139-155 (1995).

13. Mikhailov, A. \& Gundersen, G. G. Cell Motil. Cytoskel. 41, 325340 (1998).

14. Liao, G., Nagasaki, T. \& Gundersen, G. G. J. Cell Sci. 108, 3473 3483 (1995).

15. Ren, Y. et al. J. Biol. Chem. 273, 34954-34960 (1998).

16. Glaven, J. A. et al. J. Biol. Chem. 274, 2279-2285 (1999).

17. Waterman-Storer, C. \& Salmon, E. D. J. Cell Biol. 139, 417-434 (1997).

18. Waterman-Storer, C.M. \& Salmon, E. D. Curr. Opin. Cell Biol. 11, 61-67 (1999). 